\title{
Adherence to the Mediterranean Diet and Circulating Levels of Sirtuin 4 in Obese Patients: A Novel Association
}

\author{
Luigi Barrea, ${ }^{1}$ Giovanni Tarantino, ${ }^{2}$ Carolina Di Somma, ${ }^{3}$ Giovanna Muscogiuri, ${ }^{1}$ \\ Paolo Emidio Macchia, ${ }^{4}$ Andrea Falco, ${ }^{1}$ Annamaria Colao, ${ }^{4}$ and Silvia Savastano ${ }^{4}$ \\ ${ }^{1}$ I.O.S. \& COLEMAN Srl, Acerra, 80011 Naples, Italy \\ ${ }^{2}$ Dipartimento di Medicina Clinica e Chirurgia, Federico II University Medical School of Naples, Via Sergio Pansini 5, \\ 80131 Naples, Italy \\ ${ }^{3}$ IRCCS SDN, Napoli Via Gianturco 113, 80143 Naples, Italy \\ ${ }^{4}$ Dipartimento di Medicina Clinica e Chirurgia, Unit of Endocrinology, Federico II University Medical School of Naples, \\ Via Sergio Pansini 5, 80131 Naples, Italy
}

Correspondence should be addressed to Giovanni Tarantino; tarantin@unina.it

Received 6 December 2016; Accepted 16 March 2017; Published 15 June 2017

Academic Editor: Massimo Collino

Copyright (C) 2017 Luigi Barrea et al. This is an open access article distributed under the Creative Commons Attribution License, which permits unrestricted use, distribution, and reproduction in any medium, provided the original work is properly cited.

Purpose. This study was aimed at evaluating sirtuin 4 (Sirt4) levels in obese individuals, in relation to their adherence to the Mediterranean diet (MD), a healthy dietary pattern characterized by high antioxidant capacity, and markers of visceral fat storage. Subjects/Methods. Forty-three obese patients (44\% males; BMI: $36.7-58.8 \mathrm{~kg} / \mathrm{m}^{2}$ ) were consecutively included. PREvención con DIeta MEDiterránea (PREDIMED) and the 7-day food records were used to assess the adherence to MD and dietary pattern, respectively. Visceral adiposity index (VAI) was calculated. Sirt4 levels were detected by ELISA method. Results. The majority of the obese participants $(62.8 \%)$ had an average adherence to MD. Compared with average adherers, low adherers had higher BMI, energy intake, and percentage of energy from lipids, mainly saturated fat and polyunsaturated fatty acids (PUFA), and lower Sirt4 levels. After adjusting for BMI, Sirt4 levels remained negatively correlated with VAI. After adjusting for total energy intake, Sirt4 levels remained negatively associated with PREDIMED and consumption of n-3 PUFA, vitamins C and E. The threshold value of PREDIMED predicting the lowest decrease in Sirt4 levels was found at a score of 6. Conclusions. Less reduced Sirt4 levels in obese patients adhering to MD suggest a further aspect of the antioxidant advantage of MD.

\section{Introduction}

Diet, a modifiable environmental factor, is a mainstay in the management of obese individuals. Mediterranean diet (MD) is a healthy dietary pattern based on a common dietary pattern of the Mediterranean countries [1]. In particular, the MD is characterized by very low amounts of saturated fatty acid (SFA) and a high consumption of polyunsaturated fatty acid (PUFA) and micronutrients, including dietary vitamins and minerals, commonly reported to increase the plasma antioxidant capacity [2]. In addition, the quantity and quality of dietary lipids are known to have an impact on excess storage of lipid as triglycerides (TG) in both adipose tissue and in nonadipose tissue sites, such as the liver [3], which can have subsequent deleterious metabolic effects. On the other hand, the excess storage of lipid occurs as a consequence of continuous oversupply of fatty acids caused by enhanced lipolysis or adipocyte dysfunction, together with an alteration of the fatty acid oxidation in the mitochondria and the production of the reactive oxygen species (ROS), a by-product of oxidative phosphorylation $[4,5]$. In particular, an altered fatty acid oxidation pathway plays a key role in the development of hepatic steatosis (HS) and inflammation on a high-fat diet [6]. The visceral adiposity index (VAI) is a gender-specific mathematical index based on simple anthropometric [body mass index (BMI) and waist circumference (WC)] and metabolic [TG and high-density lipoprotein (HDL) cholesterol] parameters 
[7]. VAI has been proposed as a surrogate marker of adipose tissue distribution and/or dysfunction, independently correlated with insulin sensitivity and cardiometabolic risk both in the general population [8] and in patients with different pathologic conditions, including HS [9].

Sirtuins (Sirt), generally known as lysine deacetylases, are a complex of proteins involved in the control of several biological processes, including lipid oxidative metabolism [10], via the regulation of proteins' acetylation-deacetylation pathways. In turn, Sirt pathways, acting as metabolic sensors in situations of energy stress and nutrient deprivation, are regulated by macronutrient availability [11]. Despite conserved deacetylase domains, mitochondrial sirtuin 4 (Sirt4) is the most efficient lipoamidase among mitochondrial sirtuins. Sirt4 regulates the pyruvate dehydrogenase complex via enzymatic hydrolysis of the lipoamide cofactor, thus modulating acetyl coenzyme A production, Krebs cycle activity, and ROS generation [12]. Afterwards, it became apparent that Sirt4 is involved in the regulation of fatty acid oxidation in the liver [13-15]. In line with this finding, we have reported that circulating levels of Sirt 4 were reduced in obese patients with HS, although the possible contribution of the diet and the adipocyte dysfunction on this association was not investigated [16].

The current study is based on the hypothesis that the antioxidant properties of MD might be associated with the circulating levels of Sirt4 in the obese individuals. To clarify these associations, we investigated the possible relationship between circulating levels of Sirt4 and as follows: (i) the adherence to the MD; (ii) the single dietary components evaluated by 7-day food records; and (iii) degree of HS, measured by liver ultrasound and VAI, as marker of adipocyte dysfunction.

\section{Subjects and Methods}

2.1. Design and Setting. This is a cross-sectional observational study carried out by the Endocrinology Unit of the Department of Clinical Medicine and Surgery at University Federico II in Naples (Italy). The study has been performed in accordance with the Code of Ethics of the World Medical Association (Declaration of Helsinki) for experiments involving humans, and it has been approved by the Ethical Committee of the University Federico II (n.5/14). The purpose of the study was explained to both the patients and the healthy controls, and written informed consent was obtained. The study was conducted without any sponsorship. This cross-sectional observational study was registered at clinicaltrials.gov (NCT02840968).

2.2. Population Study. Out of the 257 consecutive obese subjects visiting the outpatient Obesity Unit of the Section of Endocrinology, University of Naples Federico II, from December 2013 to September 2015, 43 adult individuals referred to our unit for bariatric surgery evaluation and were enrolled in this cross-sectional observational study. A full medical history, including drug use, was collected. Criteria for exclusion from the study were as follows: (a) hypocaloric diet in the last three months or specific nutritional regimens, including vegan or vegetarian diets (38 and two subjects, resp.); (b) the presence of liver or renal failure, cancer, and acute or chronic inflammatory diseases based on a complete medical examination and laboratory investigations (seven subjects with psoriasis, three with rheumatoid arthritis, and seven with chronic obstructive pulmonary disease); (c) occasional or current use of drugs affecting $\beta$-oxidation, including nonsteroidal antiinflammatory drugs (18 subjects), $\alpha$-lipoic acid (two subjects), and valproic acid (three subjects); (d) use of weight loss medication (21 subjects) or lipid-lowering drugs (23 subjects); (e) altered thyroid hormone function tests or thyroid hormone treatment or altered somatotropic axis (50 subjects); (f) alcohol abuse according to the Diagnostic and Statistical Manual of Mental Disorders- (DSM-) V diagnostic criteria (two subjects); and (g) vitamin/mineral or antioxidant supplementation (32 subjects). Finally, six subjects dropped out from the study since they refused to undergo full laboratory-instrumental examinations (Figure 1).

Information on smoking habit and physical activity was obtained by a standard questionnaire. Subjects smoking at least one cigarette per day were considered current smokers, while former smokers were the subjects who stopped smoking at least one year before the interview. Remaining participants were defined as noncurrent smokers. Physical activity levels were expressed according to whether the participant habitually engaged at least $30 \mathrm{~min} /$ day of aerobic exercise (YES/NO). Physical active participants were advised to avoid the physical activity in the 3 days before blood sampling. Participants were classified according to their alcohol intake into two groups: YES or NO alcohol consumption. Among alcohol consumers, heavy alcohol consumption was defined as more than two standard drinks per day, which is equal to a daily intake of $<30 \mathrm{~g}$ of alcohol for men and $<20 \mathrm{~g}$ of alcohol for women.

In all individuals, systolic (SBP) and diastolic (DBP) blood pressure were measured in three times, two minutes apart, with a random zero sphygmomanometer (Gelman Hawksley Ltd., Sussex, UK) after the subject had been sitting for at least $10 \mathrm{~min}$. The average of the second and third reading was recorded.

Hypertension was defined as $\mathrm{SBP} \geq 140 \mathrm{mmHg}$ or $\mathrm{DBP} \geq 90 \mathrm{mmHg}$ on two different occasions or taking antihypertensive medication. Hypercholesterolemia was defined as a fasting blood total cholesterol level $\geq 190 \mathrm{mg} / \mathrm{dL}$ or use of lipid-lowering medication, hypertriglyceridaemia as fasting blood TG levels $\geq 150 \mathrm{mg} / \mathrm{dL}$ or use of lipid-lowering medication, and low HDL cholesterol was defined as $<40 \mathrm{mg} / \mathrm{dL}$ in men and $<50 \mathrm{mg} / \mathrm{dL}$ in women [17]. A history of using oral hypoglycemic agents or a type 2 diabetes was diagnosed according to American Diabetes Association (ADA) criteria [18].

2.3. Anthropometric Measurements. All anthropometric measurements were taken with subjects wearing only light clothes and without shoes. In each subject, BMI was calculated as weight $(\mathrm{kg}) /$ height $\left(\mathrm{m}^{2}\right)$. Height was measured to the nearest one $\mathrm{cm}$ using a wall-mounted stadiometer. Body weight was determined to the nearest $100 \mathrm{~g}$ using a calibrated balance beam scale. The degree of obesity was established on the basis of BMI cut-off points of $30.0-34.9 \mathrm{~kg} / \mathrm{m}^{2}$ (class I 


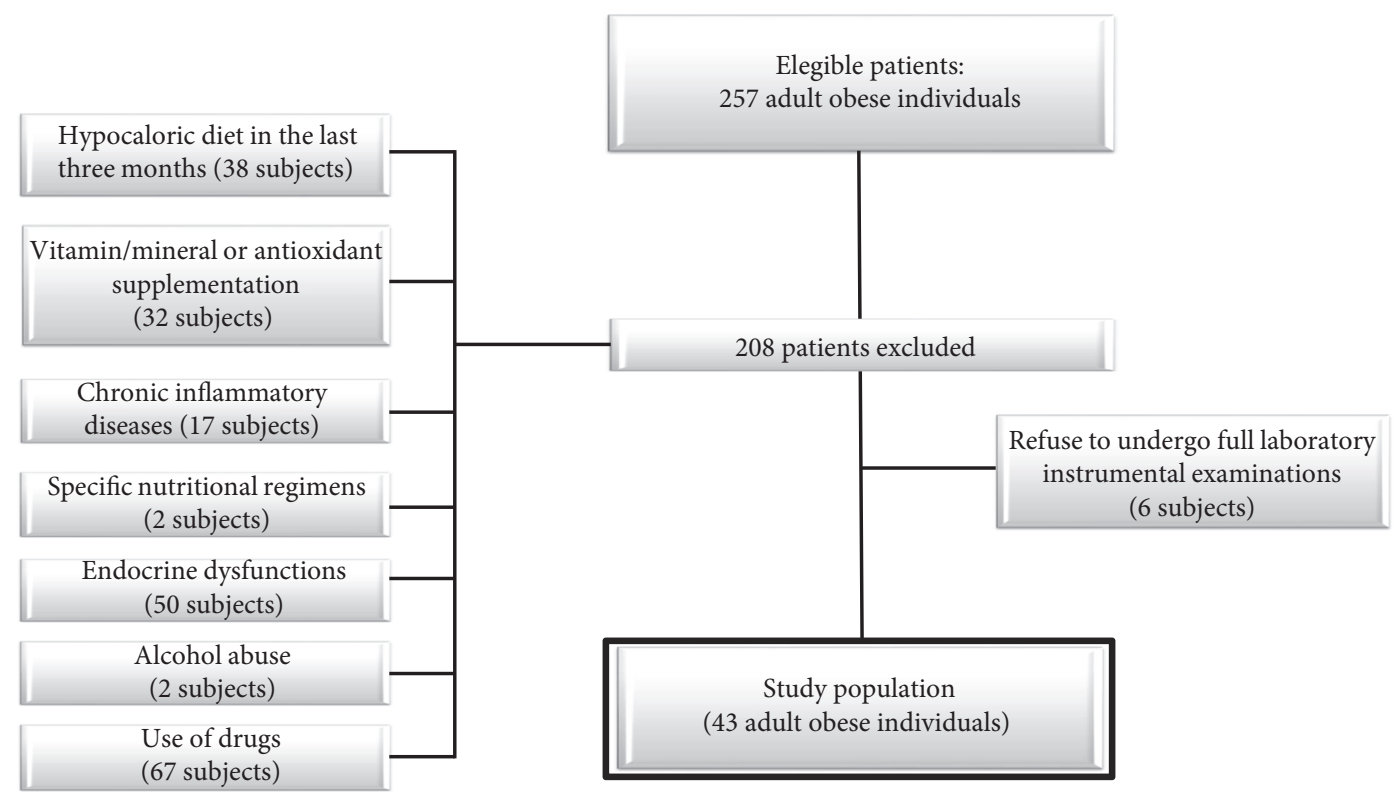

Figure 1: Flow chart of the study design.

obesity), $35.0-39.9 \mathrm{~kg} / \mathrm{m}^{2}$ (class II obesity), and $\geq 40.0 \mathrm{~kg} / \mathrm{m}^{2}$ (class III obesity) [19]. WC was measured to the closest $0.1 \mathrm{~cm}$ with a nonextensible tape. The measurements were made with the subject standing upright, feet together, and arms hanging freely at the sides, with the subjects standing and breathing normally. WC was measured at the midpoint between the inferior costal margin and the upper iliac crest. According to the National Cholesterol Education Program's Adult Treatment Panel III (NCEP-ATP III) criteria, abdominal obesity was defined as $\mathrm{WC} \geq 102 \mathrm{~cm}$ in men and $\geq 88 \mathrm{~cm}$ in women [20].

2.4. Adherence to the Mediterranean Diet. The adherence to the MD was evaluated using the previously validated 14item questionnaire for the assessment of PREvención con DIeta MEDiterránea (PREDIMED) [21]. A qualified nutritionist administered the questionnaire during a face-to-face interview to all the enrolled subjects. Briefly, for each item, scores 1 and 0 were assigned; PREDIMED score was calculated as follows: 0-5, lowest adherence; score 6-9, average adherence; and score $\geq 10$, highest adherence [21].

2.5. Dietary Assessment. As already reported by [22], data were obtained during a face-to-face interview between the patient and a qualified nutritionist. In detail, the dietary interview allowed to quantify foods and drinks by using a photographic food atlas ( $\approx 1000$ photographs) of known portion sizes to ensure accurate completion of the records [23]. Moreover, dietary data, including beverage intakes and alcohol consumption, were collected by a 7-day food records. The subjects returned the records to the nutritionist who asked supplemental questions if necessary. Data were stored and processed using a commercial software (Terapia Alimentare Dietosystem ${ }^{\circledR}$ DS-Medica, http://www. dsmedica.info). Considering quantities and qualities of foods consumed, the software is able to calculate not only the daily caloric intake but also the quantities of macronutrients (protein; total, complex, and simple carbohydrates; total fat, SFA, monounsaturated fatty acids (MUFA), PUFA: n-6 PUFA, n-3 PUFA, and n-6/n-3 PUFAs ratio; cholesterol; and fibers) and antioxidant nutrient intake, such as vitamins A, C, D, and E, and iron, selenium, and zinc. Using the same commercial software, we calculated also the Mediterranean adequacy index (MAI), obtained by dividing the sum of the percentages of dietary energy from food groups typical of a healthy reference MD, by the sum of the percentages of dietary energy of food groups that are not characteristic of a healthy reference MD [24]:

$$
\mathrm{MAI}=\frac{(\% \text { en cereals }+ \text { legumes }+ \text { potatoes }+ \text { vegetables }+ \text { fruit fresh and dry }+\mathrm{fish}+\text { wine }+ \text { virgin olive oil })}{(\% \text { en milk }+ \text { cheese }+ \text { meat }+ \text { eggs }+ \text { animal fats and margarines }+ \text { sweet beverages }+ \text { cakes } / \text { pies }+ \text { cookies })} .
$$

The quality was assessed using the Index of Nutritional Quality (INQ), which is based on the nutritional density per $1000 \mathrm{kcal}$. The INQ was calculated by dividing the nutritional intake per $1000 \mathrm{kcal}$ of total energy intake by the recommended intake (RI) of protein, carbohydrate, and fat per $1000 \mathrm{kcal}$. The RI values were obtained from the MD recommended, in particular, the macronutrient energy recognized as adequate in the $\mathrm{MD}$ is $55-60 \%$ of carbohydrates, $10-15 \%$ of proteins, and 
$25-30 \%$ fat [25]. Each INQ value $\geq 1.0$ indicates adequate nutritional intake, while values $<1.0$ indicated inadequate nutritional status. In this study, the INQ was calculated for protein, carbohydrate, and fat [26].

2.6. Assay Methods. Samples were collected in the morning between 8 and 10 a.m., after an overnight fast of at least $8 \mathrm{~h}$ and stored at $-80^{\circ} \mathrm{C}$ until being processed. All biochemical analyses including fasting plasma glucose, total cholesterol, fasting plasma TG, alanine transaminase (ALT), aspartate aminotransferase (AST), and $\gamma$-glutamyltransferase $(\gamma \mathrm{GT})$ were performed with a Roche Modular Analytics System in the Central Biochemistry Laboratory of our institution. Low-density lipoprotein (LDL) cholesterol and HDL cholesterol were determined by a direct method (homogeneous enzymatic assay for the direct quantitative determination of LDL and HDL cholesterol). Fasting insulin levels were measured by a solid-phase chemiluminescent enzyme immunoassay using a commercially available kits (Immunolite Diagnostic Products Co, Los Angeles, CA). The intra-assay coefficients of variations $(\mathrm{CV})$ were $<5.5 \%$.

2.7. Metabolic Indices. Homeostasis model assessment-insulin resistance (HoMA-IR) was calculated according to Matthews et al. [27]: a value of HoMA - IR > 2.5 was used as cutoff of insulin resistance. VAI score has been calculated by the following sex-specific formula, with TG levels expressed in $\mathrm{mmol} / \mathrm{L}$ and HDL levels expressed in $\mathrm{mmol} / \mathrm{L}$ :

$$
\begin{aligned}
\text { Males: VAI }= & \left(\frac{\mathrm{WC}}{39.68+(1.88 \times \mathrm{BMI})}\right) \\
& \times\left(\frac{\mathrm{TG}}{1.03}\right) \times\left(\frac{1.31}{\mathrm{HDL}}\right), \\
\text { Females: VAI }= & \left(\frac{\mathrm{WC}}{36.58+(1.89 \times \mathrm{BMI})}\right) \\
& \times\left(\frac{\mathrm{TG}}{0.81}\right) \times\left(\frac{1.52}{\mathrm{HDL}}\right) .
\end{aligned}
$$

Age-specific VAI cut-off values were used according to Amato et al. $[8,28]$. In details, cutoffs in subjects aged $\leq 30$, $31-42,43-52$, and 53-66 years were 2.52, 2.23, 1.92, 1.93, respectively $[8,28]$.

2.8. Determination of Circulating Levels of Sirt4. For the determination of circulating levels of Sirt4, venous blood samples from the antecubital vein were obtained following standard procedures. Blood was drawn into a $9 \mathrm{~mL}$ serum tube. Samples were centrifuged for $10 \mathrm{~min}$ at relative centrifugal force (RCF) of 850-1000. A part of sera was collected, aliquoted, and frozen at $-20^{\circ} \mathrm{C}$ for the successive determination of circulating levels of Sirt4. The kit for in vitro quantitative measurement of Sirt4 in human tissue homogenates and other biological fluids, based on a sandwich enzyme immunoassay, was provided by Uscn Life Science \& Technology Company, 3603 Double Lake Dr, Missouri City, TX 77459. The detection range was $0.156-10 \mathrm{ng} / \mathrm{mL}$, as reported elsewhere [16]. Briefly, the standard curve concentrations used for the ELISA were $10 \mathrm{ng} / \mathrm{mL}, 5 \mathrm{ng} / \mathrm{mL}, 2.5 \mathrm{ng} / \mathrm{mL}, 1.25 \mathrm{ng} / \mathrm{mL}$, $0.625 \mathrm{ng} / \mathrm{mL}, 0.312 \mathrm{ng} / \mathrm{mL}$, and $0.156 \mathrm{ng} / \mathrm{mL}$. The sensitivity of this assay was defined as the lowest protein concentration that could be differentiated from zero. It was determined by adding two standard deviations to the mean optical density value of twenty zero standard replicates and calculating the corresponding concentration. The minimum detectable dose of human Sirt4 was less than $0.051 \mathrm{ng} / \mathrm{mL}$. No significant cross-reactivity or interference between human Sirt4 and other sirtuins was observed, according to manufacturer data. Intra-assay precision was determined as follows: three samples with low, middle, and high level human Sirt4 were tested 20 times on one plate. The inter-assay precision was weighed testing three samples with low, middle, and high level human Sirt 4 on 3 different plates, 8 replicates in each plate. The coefficient of variation, calculated by $\mathrm{SD} /$ mean $\tilde{n} 100$ for the intra-assay and inter-assay was $<10 \%$ and $<12 \%$, respectively.

2.9. HS Evaluation. The presence of HS, commonly known as "bright liver", was assessed with a US diagnostic system (Logiq P5, General Electric, Milan, Italy) with a 3.5 MHz convex probe. All the US determinations were made by the same trained operator. The intraoperator variability, as evaluated in 20 subjects within 1 week from the first ultrasonographic examination, showed an overall $r$ value of $0.92(p<0.001)$.

The classification of "bright liver" or HS was based on the following scale of hyperechogenicity: grade $0=$ absent, grade 1 = light, grade $2=$ moderate, and grade $3=$ severe, pointing out the difference between the densities of the liver and the right kidney [29]. Technically, echo intensity can be influenced by many factors, particularly by gain intensity. To avoid confounding factors that could modify echo intensity and thus bias comparisons, mean brightness levels of both liver and right kidney cortex were obtained on the same longitudinal sonographic plane.

2.10. Statistical Methods. The minimum required total sample was calculated using the pooled standard deviation with the level test 0.05 and power $90 \%$ of means the circulating levels of Sirt 4 in obese patients. With a type I error of 0.10 and a type of II $(\beta)$ error of 0.05 , the resulting size was 13 subjects.

Results are expressed as mean \pm SD or as median plus range according to the variable's distributions evaluated by Kolmogorov-Smirnov test. To correct for skewed distributions, circulating levels of Sirt4 were logarithmically transformed and back-transformed for presentation in tables and figures.

Differences between two groups were analyzed by MannWhitney $U$ test or Student's unpaired $t$-test, when appropriate. Differences among three groups were analyzed by the Kruskal-Wallis rank test or ANOVA test, with the Bonferroni test as post hoc test, according to the variable's distribution. The $\mathrm{chi}^{2}\left(\chi^{2}\right)$ test was used to test the significance of differences in frequency distributions. The correlations between variables were performed using Spearman's rho correlation coefficients. Multinomial logistic regression was performed to model the relationship between the circulating levels of Sirt4 and the three groups of HS classification (grade 1, grade 2, and grade 3).

Bivariate proportional odds ratio (OR) models and 95\% interval confidence (IC) were performed to assess the 
TABLE 1: Circulating levels of Sirt4 in the study population according to gender, obesity-related morbidities, cardiometabolic indices, HS, and major lifestyle factors.

\begin{tabular}{|c|c|c|c|}
\hline \multirow[t]{2}{*}{ Parameters } & & \multicolumn{2}{|c|}{$\begin{array}{l}\text { Circulating levels of Sirt4 (ng/mL) } \\
n=43\end{array}$} \\
\hline & & Median (min-max) & $p$ value \\
\hline \multirow{2}{*}{ Gender } & Males & $0.37(0.10-2.75)$ & \multirow{2}{*}{0.406} \\
\hline & Females & $0.68(0.11-5.56)$ & \\
\hline \multirow{2}{*}{ Hypertension } & Yes & $0.15(0.10-0.42)$ & \multirow{2}{*}{$<0.001$} \\
\hline & No & $1.22(0.11-5.56)$ & \\
\hline \multirow{2}{*}{ Hypercholesterolaemia } & Yes & $0.28(0.11-2.51)$ & \multirow{2}{*}{0.015} \\
\hline & No & $0.24(0.10-5.56)$ & \\
\hline \multirow{2}{*}{ Hypertriglyceridaemia } & Yes & $0.28(0.11-2.39)$ & \multirow{2}{*}{0.018} \\
\hline & No & $1.30(0.10-5.56)$ & \\
\hline \multirow{2}{*}{ HDL cholesterol } & <cutoff & $0.18(0.10-1.26)$ & \multirow{2}{*}{$<0.001$} \\
\hline & $>$ cutoff & $1.88(0.16-5.56)$ & \\
\hline \multirow{2}{*}{ Type 2 diabetes } & Yes & $0.15(0.10-1.11)$ & \multirow{2}{*}{0.001} \\
\hline & No & $0.97(0.11-5.56)$ & \\
\hline \multirow{2}{*}{ VAI } & $>$ cutoff & $0.29(0.10-2.66)$ & \multirow{2}{*}{$<0.001$} \\
\hline & <cutoff & $3.23(1.22-5.56)$ & \\
\hline \multirow{3}{*}{ HS } & Grade 1 (mild) & $3.79(1.33-5.56)$ & \multirow{3}{*}{0.007} \\
\hline & Grade 2 (moderate) & $2.80(1.66-3.42)$ & \\
\hline & Grade 3 (severe) & $0.24(0.18-0.37)$ & \\
\hline \multirow{2}{*}{ Current smokers } & Yes & $0.16(0.10-1.22)$ & \multirow{2}{*}{0.001} \\
\hline & No & $1.26(0.11-5.56)$ & \\
\hline \multirow{2}{*}{ Physical activity } & No & $0.29(0.10-2.51)$ & \multirow{2}{*}{0.001} \\
\hline & Yes & $2.75(0.11-5.56)$ & \\
\hline \multirow{2}{*}{ Alcohol consumption } & Yes & $1.94(0.10-3.42)$ & \multirow{2}{*}{0.190} \\
\hline & No & $0.38(0.11-5.56)$ & \\
\hline
\end{tabular}

Circulating levels of Sirt4 were lower in individuals with obesity-related comorbidities, adiposity dysfunction, HS, current smokers, and physically inactive individuals. As HoMA-IR and FLI were increased in all the study population, these variables were not included in this analysis. Circulating levels of Sirt4 were significantly different in the presence of obesity-related comorbidities, adiposity dysfunction, HS, and among current smokers or physically inactive individuals, while there were no significant differences according to gender and alcohol consumption. Results are expressed as median plus range according to variable distributions evaluated by Kolmogorov-Smirnov test. Differences between two groups were analyzed by Mann-Whitney $U$ test. Differences among HS grades were analyzed by the Kruskal-Wallis rank test followed by Bonferroni post hoc test. A $p$ value in bold type denotes a significant difference $(p<0.05)$. Hypertension was defined as $\mathrm{SBP} \geq 140 \mathrm{mmHg}$ or $\mathrm{DBP} \geq 90 \mathrm{mmHg}$ on two different occasions or taking antihypertensive medication. Hypercholesterolemia was defined as a fasting blood total cholesterol level $\geq 190 \mathrm{mg} / \mathrm{dL}$ or use of lipid-lowering medication, hypertriglyceridaemia was defined as fasting blood triglyceride levels $\geq 150 \mathrm{mg} / \mathrm{dL}$ or use of lipid-lowering medication, and low HDL cholesterol was defined as $<40 \mathrm{mg} / \mathrm{dL}$ in men and $<50 \mathrm{mg} / \mathrm{dL}$ in women [17]. A history of using oral hypoglycemic agents or a type 2 diabetes was diagnosed according to American Diabetes Association (ADA) criteria [18]. Age-specific VAI cut-off values were used according to Amato et al. [8,25]. In detail, cutoffs in subjects aged $\leq 30,31-42,43-52$, and 53-66 years were $2.52,2.23,1.92$, and 1.93 , respectively $[8,25]$. The classification of "bright liver" or HS was based on the following scale of hyperechogenicity: grade $0=$ absent, grade $1=$ light, grade $2=$ moderate, and grade $3=$ severe, pointing out the difference between the densities of the liver and the right kidney [26]. Subjects smoking at least one cigarette per day were considered current smokers. Physical activity levels were expressed according to whether the participant habitually engaged at least $30 \mathrm{~min} /$ day of aerobic exercise. Among alcohol consumers, heavy alcohol consumption was defined as more than two standard drinks per day, which is equal to a daily intake of $<30 \mathrm{~g}$ of alcohol for men and $<20 \mathrm{~g}$ of alcohol for women. Sirt4: sirtuin 4; HDL: high-density lipoprotein; VAI: visceral adiposity index; HS: hepatic steatosis.

association among quantitative variables (all food items of the PREDIMED questionnaire and PREDIMED score). A multiple linear regression model with ordinal predictors was designed to identify critical threshold values $(m)$, expressed as $R^{2}$, beta $(\beta)$, and $t$, of the PREDIMED score at which significant changes occur in the response circulating levels of Sirt4, as follows:

$$
\text { Circulating levels of Sirt } 4_{i}=\beta_{0}+\left(\beta_{1} \text { PREDIMED score }_{i 1}+\cdots+\beta_{m-1} \text { PREDIMED score }_{i, m-1}\right)+\varepsilon_{i} \text {. }
$$

For the calculation of ODDS, circulating levels of Sirt4 were dichotomized in two categories, below and above the median (median of circulating levels of Sirt 4 was $0.42 \mathrm{ng} / \mathrm{mL}$ ). The calculation of OR was obtained of bivariate regression analysis model. 
In addition, two multiple linear regression analysis models (stepwise method), expressed as $R^{2}$, beta $(\beta)$, and $t$, with circulating levels of Sirt4 as dependent variables were used to estimate the predictive value of the following: (a) all food items of PREDIMED questionnaire and PREDIMED score and (b) total energy, SFA, n-3 PUFA, vitamin C, and vitamin E. In these analyses, we entered only those variables that had a $p$ value $<0.05$ in the univariate analysis (partial correlation). To avoid multicollinearity, variables with a variance inflation factor (VIP) $>10$ were excluded. Values $\leq 5 \%$ were considered statistically significant. Data were stored and analyzed using the MedCalc ${ }^{\circledR}$ package (Version 12.3.0 1993-2012 MedCalc Software bvba-MedCalc Software, Mariakerke, Belgium). Multinomial logistic regression, bivariate proportional OR model, and multiple linear regression model were carried out using the $\mathrm{R}$ Project for Statistical Computing 2014 (http://www.R-project.org).

\section{Results}

All participants to the study completed the PREDIMED questionnaires and the 7-day food records. Study population consisted of 43 obese patients, aged 25-53 years (44\%, 19 males). BMIs ranged from 36.7 to $58.8 \mathrm{~kg} / \mathrm{m}^{2}(88.4 \%$, 38 subjects were class III-obesity), $27.9 \%$ (12 subjects) were hypertensive, and $20.9 \%$ (9 subjects) had diagnosis of type 2 diabetes. Median values of HoMA-IR range from 2.5 to 15.7. Median of VAI was 5.3 (1.6-17.3). In particular, VAI was higher than sex and age-specific cutoffs in $79.1 \%$ (34 subjects). Moderate/severe HS was diagnosed in $90.7 \%$ (39 subjects). Current smokers were $32.6 \%$ (14 subjects) and alcohol consumption was reported in $30.2 \%$ (13 subjects). A moderate-intensity aerobic activity at least 5 days per week was reported in 25.6\% (11 subjects). Among physical active participants, no subject engaged more than $30 \mathrm{~min} /$ day/week.

Circulating levels of Sirt4 according to gender, obesityrelated comorbidities, metabolic indices, HS, and major lifestyle factors are reported in Table 1. As shown in the table, circulating levels of Sirt 4 were significantly different in the presence of obesity-related comorbidities, adiposity dysfunction, HS, and among current smokers or physically inactive individuals, while there were no significant differences according to gender and alcohol consumption.

Response frequency of dietary components included in the PREDIMED questionnaire of the patients is reported in Table 2. Extra virgin olive oil was the most consumed food item, followed by fish with the wine intake in third position. According to the PREDIMED score, all obese participants had a low (PREDIMED score $\leq 5$ ) or average adherence (PREDIMED score 6-9) to the MD, with no subjects reaching a score indicating a high adherence to the MD.

In particular, on the basis of the PREDIMED score, obese participants were divided into low (37.2\%) and average adherence $(62.8 \%)$. Study participants' characteristics grouped according to the PREDIMED score are summarized in Table 3. As shown in table, obese subjects with low adherence to the MD presented with much lower circulating levels of Sirt4, higher BMI, and more altered metabolic
TABLE 2: Response frequency of dietary components included in the PREDIMED questionnaire of the patients.

\begin{tabular}{lcc}
\hline Questions in PREDIMED questionnaire & $n$ & $\%$ \\
\hline (1) Use of extra virgin olive oil as main culinary lipid & 33 & 76.7 \\
(2) Extra virgin olive oil $>$ 4 tablespoons & 22 & 51.2 \\
(3) Vegetables $\geq 2$ servings/day & 21 & 48.8 \\
(4) Fruits $\geq 3$ servings/day & 18 & 41.9 \\
(5) Red/processed meats < 1/day & 21 & 48.8 \\
(6) Butter, cream, margarine < 1/day & 19 & 44.2 \\
(7) Soda drinks $<1 /$ day & 10 & 23.3 \\
(8) Wine glasses $\geq 7 /$ week & 24 & 55.8 \\
(9) Legumes $\geq 3 /$ week & 21 & 48.8 \\
(10) Fish/seafood $\geq 3 /$ week & 25 & 58.1 \\
(11) Commercial sweets and confectionery $\leq 2 /$ week & 12 & 27.9 \\
(12) Tree nuts $\geq 3 /$ week & 13 & 30.2 \\
(13) Poultry more than red meats & 21 & 48.8 \\
(14) Use of sofrito sauce $\geq 2 /$ week & 23 & 53.5 \\
\hline
\end{tabular}

Extra virgin olive oil was the most consumed food item, followed by fish and wine intake. Results are expressed as percentage of response obtained with PREDIMED questionnaire. PREDIMED: PREvención con DIetaMEDiterránea ([20] in the text).

indices than average adherers. No differences were observed in age and male-to-female ratio.

In Table 4, we reported the total energy and the daily macronutrients/micronutrients intake obtained from the 7-day food records. As shown in the table, obese individuals with low MD adherence had significantly higher energy intake and percentage of energy from lipids, mainly SFA and PUFA, and a significantly lower fiber intake than average adherers. In addition, low adherers consumed significantly lower complex carbohydrates and antioxidant nutrient intake than average adherers, including n-3 PUFA, A, C, and $\mathrm{E}$ vitamins, and micronutrients. In addition, as expected, obese individuals with low MD adherence presented INQ $<1$ for protein, carbohydrate, and fat and lower MAI compared with average adherers.

3.1. Correlation Studies. The correlations among circulating levels of Sirt4, anthropometric measurements, and metabolic parameters are summarized in Table 5. In Table 6, the multinomial logistic regression of circulating levels of Sirt 4 with HS grade are reported. The highest circulating levels of Sirt4 were associated with the lowest odds of HS (grade 1), $p=0.002, R^{2}=0.861$, and $\mathrm{AIC}=56.623$.

The results of bivariate proportional odds ratio model performed to assess the association of circulating levels of Sirt4 with food items of PREDIMED questionnaire, and PREDIMED score are reported in Table 7. In obese patients, the lowest decrease in circulating levels of Sirt4 was significantly associated with the highest odds of consumption of the Mediterranean food items, in particular fruits $(p=0.002)$, extra virgin olive oil $(p=0.004)$, vegetables $(p=0.008)$, fish $(p=0.011)$, and wine $(p=0.018)$, and with highest score of adherence of $\mathrm{MD}(p=0.004)$. On the contrary, the highest decrease in circulating levels of Sirt4 was significantly associated with the highest odds of consumption 
TABLE 3: Sociodemographic and anthropometric characteristics, metabolic profile, and circulating levels of Sirt4 in obese individuals grouped on the basis of the adherence to the Mediterranean diet.

\begin{tabular}{|c|c|c|c|}
\hline Parameters & $\begin{array}{c}\text { Obese } \\
\text { Low adherence MD } \\
n=16\end{array}$ & $\begin{array}{c}\text { Obese } \\
\text { Average adherence MD } \\
n=27\end{array}$ & $p$ value \\
\hline Age (years) & $38.1 \pm 3.4$ & $35.9 \pm 7.7$ & 0.356 \\
\hline Gender $\mathrm{M} / \mathrm{F}$ & $8 / 8$ & $11 / 16$ & 0.784 \\
\hline \multicolumn{4}{|l|}{ Anthropometric measurement } \\
\hline BMI $\left(\mathrm{kg} / \mathrm{m}^{2}\right)$ & $52.0 \pm 3.4$ & $42.4 \pm 2.9$ & $<0.001$ \\
\hline WC males $(\mathrm{cm})$ & $151.1 \pm 9.8$ & $136.8 \pm 11.6$ & 0.012 \\
\hline WC females $(\mathrm{cm})$ & $144.4 \pm 17.1$ & $125.4 \pm 15.4$ & 0.012 \\
\hline \multicolumn{4}{|l|}{ Metabolic profile } \\
\hline Circulating levels of Sirt4 (ng/mL) & $0.16(0.10-0.29)$ & $1.33(0.11-5.56)$ & $<0.001$ \\
\hline SBP (mmHg) & $136.0 \pm 12.4$ & $127.4 \pm 10.7$ & 0.022 \\
\hline DBP (mmHg) & $85.0(70.0-100.0)$ & $80.0(60.0-95.0)$ & 0.090 \\
\hline Fasting glucose $(\mathrm{mg} / \mathrm{dL})$ & $109.0(73.0-143.0)$ & $94.0(68.0-193.0)$ & 0.004 \\
\hline Insulin $(\mu \mathrm{U} / \mathrm{mL})$ & $36.1 \pm 7.9$ & $26.7 \pm 8.5$ & 0.001 \\
\hline Total cholesterol (mg/dL) & $211.1 \pm 31.9$ & $179.4 \pm 27.4$ & 0.003 \\
\hline HDL cholesterol (mg/dL) & $36.8 \pm 6.1$ & $51.3 \pm 11.1$ & $<0.001$ \\
\hline LDL cholesterol (mg/dL) & $134.9 \pm 23.0$ & $102.8 \pm 26.5$ & $<0.001$ \\
\hline Triglycerides (mg/dL) & $171.5(89.0-380.0)$ & $101.0(49.0-283.0)$ & 0.015 \\
\hline $\operatorname{ALT}(\mathrm{U} / \mathrm{L})$ & $38.0(12.0-91.0)$ & $32.0(13.0-99.0)$ & 0.439 \\
\hline AST (U/L) & $25.0(12.0-52.0)$ & $23.0(10.0-91.0)$ & 0.793 \\
\hline$\gamma \mathrm{GT}(\mathrm{U} / \mathrm{L})$ & $30.0(12.0-61.0)$ & $30.5(14.0-101.0)$ & 0.776 \\
\hline \multicolumn{4}{|l|}{ Metabolic indices } \\
\hline HoMA-IR & $10.0 \pm 3.0$ & $6.4 \pm 2.4$ & $<0.001$ \\
\hline VAI & $8.5(3.3-17.3)$ & $4.7(1.6-15.8)$ & 0.003 \\
\hline \multicolumn{4}{|l|}{ HS evaluation } \\
\hline HS (grade 1/2/3) & $0 / 1 / 15$ & $4 / 9 / 14$ & $<0.001$ \\
\hline FLI & $99.9(98.8-100.0)$ & 98.7 (80.3-99.9) & $<0.001$ \\
\hline
\end{tabular}

Obese individuals with low adherence to the MD exhibited statistically significant differences in anthropometric measurements, metabolic profile, metabolic indices, and HS evaluation compared with average adherer counterpart. No differences were observed in age and male-to-female ratio. Results are expressed as mean \pm standard deviation or as median plus range according to variable distributions evaluated by Kolmogorov-Smirnov test. Differences between groups were analyzed by unpaired Student's $t$-test or Wilcoxon signed-rank test, when appropriate. The $\operatorname{chi}^{2}\left(\chi^{2}\right)$ test was used to test the significance of differences between the two groups. $p$ value indicates comparison between those with low adherence versus those with average adherence to the MD. A $p$ value in bold type denotes a significant difference $(p<0.05)$. MD: Mediterranean diet; BMI: body mass index; WC: waist circumference; Sirt4: sirtuin 4; SBP: systolic blood pressure; DBP: diastolic blood pressure; HDL: high-density lipoprotein; LDL: low-density lipoprotein; ALT: alanine aminotransferase; AST: aspartate aminotransferase; $\gamma \mathrm{GT}$ : gamma glutamyl transferase: HoMA-IR: homeostatic model assessment-insulin resistance; VAI: visceral adiposity index; HS: hepatic steatosis; FLI: fatty liver index.

of butter $(p=0.015)$. In Table 8 , the correlations among circulating levels of Sirt4, PREDIMED score, total energy and daily macronutrients/micronutrients intake, INQ, and MAI evaluated by using the 7-day food records are reported. As expected, INQ and MAI showed a highly positive correlation with PREDIMED score (INQ protein, $r=0.793$; INQ carbohydrates, $r=0.824$; INQ fat, $r=0.846$; MAI, $r=0.974$; $p<0.001$, respectively). Circulating levels of Sirt4 correlated with PREDIMED score and MAI $(p<0.001)$, with all the dietary macronutrients and micronutrients, except for total carbohydrates and PUFA $(p=0.430)$ and INQ for each macronutrient $(p<0.001)$. After adjusting for total energy intake, all the associations were lost, except for PREDIMED score $(p=0.021)$, INQ protein $(p=0.003)$, the consumption of fat SFA $(p=0.016)$ and n-3 PUFA $(p<0.001)$, and the intake of vitamins $\mathrm{C}$ and $\mathrm{E} \quad(p<0.001$ and $p=0.034$, respectively); as shown in Table 8 . To compare the relative predictive power of the food items included in the PREDIMED and the score of adherence to the MD associated with the circulating levels of Sirt4, we performed a multiple linear regression analysis using models that included as measures of the consumption frequency of each food items of PREDIMED questionnaire along with the PREDIMED score. Using these models, PREDIMED score entered at the first step $(p<0.001)$ and followed by the fruit consumption $\geq 3$ serving/day $(p=0.009)$. To compare the relative predictive power of the intake of food and beverage associated with the circulating levels of Sirt4, we performed a multiple linear regression analysis using models that included as measures of the intake of the single micro and macronutrients obtained by the 7-day food records. Using these models, n-3 PUFA entered at the first step $(p<0.001)$, followed by vitamin $\mathrm{E}$ 
TABLE 4: Total energy and daily macronutrient/micronutrient intake of obese individuals grouped on the basis of the adherence to the Mediterranean diet.

\begin{tabular}{|c|c|c|c|}
\hline Parameters & $\begin{array}{c}\text { Obese } \\
\text { Low adherence MD } \\
n=16 \\
\end{array}$ & $\begin{array}{c}\text { Obese } \\
\text { Average adherence MD } \\
n=27\end{array}$ & $p$ value \\
\hline Total energy (kcal) & $3473.1 \pm 271.9$ & $2822.3 \pm 287.7$ & $<0.001$ \\
\hline Protein (\% of total kcal) & $14.0(11.0-18.0)$ & $16.0(12.0-20.0)$ & 0.045 \\
\hline INQ protein & $0.98 \pm 0.03$ & $1.08 \pm 0.07$ & $<0.001$ \\
\hline Carbohydrate (\% of total kcal) & $56.0(53.0-61.0)$ & $57.0(54.0-60.0)$ & 0.469 \\
\hline INQ carbohydrate & $0.97 \pm 0.03$ & $1.03 \pm 0.03$ & $<0.001$ \\
\hline Complex (\% of total kcal) & $28.8 \pm 5.5$ & $31.8 \pm 4.0$ & 0.049 \\
\hline Simple (\% of total kcal) & $27.8 \pm 4.5$ & $25.2 \pm 4.0$ & 0.055 \\
\hline Fat (\% of total kcal) & $29.0(28.0-30.0)$ & $27.0(25.0-30.0)$ & $<0.001$ \\
\hline INQ Fat & $0.96 \pm 0.03$ & $1.06 \pm 0.05$ & $<0.001$ \\
\hline SFA (\% of total kcal) & $11.5(8.3-15.7)$ & $9.0(1.7-10.3)$ & $<0.001$ \\
\hline MUFA (\% of total kcal) & $12.8(11.2-14.3)$ & $15.7(14.5-17.7)$ & $<0.001$ \\
\hline PUFA (\% of total kcal) & $3.3(1.8-9.3)$ & $2.9(1.8-5.9)$ & 0.191 \\
\hline n-6 PUFA (g/day) & $10.9(4.8-34.7)$ & $5.7(0.4-15.4)$ & 0.001 \\
\hline n-3 PUFA (g/day) & $2.2(0.6-2.8)$ & $3.4(1.3-12.4)$ & $<0.001$ \\
\hline n-6/n-3 PUFAs ratio & $5.9(2.1-20.8)$ & $1.6(0.1-11.8)$ & $<0.001$ \\
\hline Cholesterol (mg/day) & $335.9 \pm 42.2$ & $323.0 \pm 32.2$ & 0.262 \\
\hline Fiber (g/day) & $20.4(8.2-25.0)$ & $24.0(10.1-28.1)$ & 0.021 \\
\hline Retinol Eq ( $\mu \mathrm{g} /$ day) & $756.5(451.0-1990.0)$ & $1249.0(527.0-2012.0)$ & 0.001 \\
\hline Vitamin C (mg/day) & $71.0 \pm 26.3$ & $159.0 \pm 44.7$ & $<0.001$ \\
\hline Vitamin D ( $\mu \mathrm{g} /$ day $)$ & $1.6 \pm 0.6$ & $2.1 \pm 0.7$ & 0.014 \\
\hline Vitamin E (mg/day) & $10.5 \pm 1.6$ & $16.4 \pm 2.3$ & $<0.001$ \\
\hline Iron (mg/day) & $8.2 \pm 1.9$ & $10.6 \pm 2.9$ & 0.005 \\
\hline Selenium ( $\mu \mathrm{g} /$ day $)$ & $15.2(9.0-33.9)$ & $22.1(8.7-42.5)$ & 0.003 \\
\hline Zinc (mg/day) & $8.8 \pm 1.5$ & $10.4 \pm 1.3$ & $<0.001$ \\
\hline MAI & $1.09 \pm 0.34$ & $3.18 \pm 0.81$ & $<0.001$ \\
\hline
\end{tabular}

Obese individuals with low MD adherence had a higher energy intake, a higher percentage of energy from lipids, mainly SFA and PUFA, and a lower fiber intake than average adherers. Low adherers consumed lower complex carbohydrates and lower antioxidant intake than average adherers, including n-3 PUFA, A, C, and $\mathrm{E}$ vitamins, and micronutrients. Results are expressed as mean \pm standard deviation or as median plus range according to variable distributions evaluated by Kolmogorov-Smirnov test. Differences between groups were analyzed by unpaired Student's $t$-test or Wilcoxon signed-rank test, when appropriate. $p$ value indicates comparison between those with low adherence versus those with average adherence to the MD. A $p$ value in bold type denotes a significant difference $(p<0.05)$. MD: Mediterranean diet; INQ: index of nutritional quality; SFA: saturated fatty acids; MUFA: monounsaturated fatty acids; PUFA: polyunsaturated fatty acids; MAI: Mediterranean adequacy index.

$(p<0.001)$ and vitamin C $(p=0.048)$; results are reported in Table 9.

Based on the multiple linear regression analysis with ordinal predictors, the most significant $(\alpha=0.05)$ threshold value of the PREDIMED score predicting the lowest decrease in circulating levels of Sirt4 was found at a score of $6(p<0.001)$ (Figure 2).

\section{Discussion}

The present study evidenced a positive association between adherence to the MD and circulating levels of Sirt4 in obese individuals. In addition, a careful investigation of the dietary pattern using the 7-day food records demonstrated that the circulating levels of Sirt4 are negatively correlated with energy intake, and positively with the intake of antioxidant vitamins and minerals considered in this study. Finally, we found that circulating levels of Sirt 4 were negatively correlated with surrogate markers of ectopic fat storage and visceral fat dysfunction, independently of the severity of the obesity. Of interest, although experimental studies evidenced that physical activity might affect the Sirt 4 expression [30], we found that the associations between Sirt4 and variables included in this study remained significant also after adjusting for physical activity.

We previously reported that in obese individuals with higher degree of HS, there were lower circulating levels of Sirt4 [16]. Although in our preliminary study, we hypothesized the involvement of a high calorie intake in reducing circulating levels of Sirt4; the possible influence of the dietary pattern on the circulating levels of Sirt 4 was not investigated.

The present study further extends our previous observations as it demonstrates that lower circulating levels of Sirt4 were actually associated with a specific dietary pattern in 
TABle 5: Correlations among circulating levels of Sirt4 with demographics, anthropometric measurements, metabolic profile, metabolic indices, and HS evaluation.

\begin{tabular}{|c|c|c|c|c|}
\hline \multirow{3}{*}{ Parameters } & \multicolumn{4}{|c|}{$\begin{array}{l}\text { Circulating levels of Sirt4 (ng/mL) } \\
\qquad n=43\end{array}$} \\
\hline & \multicolumn{2}{|c|}{ Simple correlation } & \multicolumn{2}{|c|}{ Adjusted for BMI } \\
\hline & $r$ & $p$ value & $r$ & $p$ value \\
\hline Age (years) & -0.363 & 0.017 & -0.249 & 0.122 \\
\hline \multicolumn{5}{|c|}{ Anthropometric measurement } \\
\hline BMI $\left(\mathrm{kg} / \mathrm{m}^{2}\right)$ & -0.804 & $<0.001$ & - & - \\
\hline $\mathrm{WC}(\mathrm{cm})$ & -0.707 & $<0.001$ & -0.339 & 0.033 \\
\hline \multicolumn{5}{|l|}{ Metabolic profile } \\
\hline $\mathrm{SBP}(\mathrm{mmHg})$ & -0.512 & $<0.001$ & 0.038 & 0.817 \\
\hline $\mathrm{DBP}(\mathrm{mmHg})$ & -0.318 & 0.038 & 0.075 & 0.645 \\
\hline Fasting glucose $(\mathrm{mg} / \mathrm{dL})$ & -0.369 & 0.015 & -0.067 & 0.679 \\
\hline Insulin $(\mu \mathrm{U} / \mathrm{mL})$ & -0.543 & $<0.001$ & -0.147 & 0.366 \\
\hline Total cholesterol (mg/dL) & -0.522 & $<0.001$ & -0.257 & 0.109 \\
\hline HDL cholesterol (mg/dL) & 0.814 & $<0.001$ & 0.434 & 0.005 \\
\hline LDL cholesterol (mg/dL) & -0.642 & $<0.001$ & -0.276 & 0.085 \\
\hline Triglycerides (mg/dL) & -0.454 & 0.002 & -0.246 & 0.126 \\
\hline $\operatorname{ALT}(\mathrm{U} / \mathrm{L})$ & 0.081 & 0.611 & 0.130 & 0.424 \\
\hline AST (U/L) & -0.015 & 0.926 & 0.041 & 0.801 \\
\hline$\gamma \mathrm{GT}(\mathrm{U} / \mathrm{L})$ & 0.117 & 0.468 & -0.006 & 0.971 \\
\hline \multicolumn{5}{|l|}{ Metabolic indices } \\
\hline HoMA-IR & -0.627 & $<0.001$ & -0.111 & 0.497 \\
\hline VAI & -0.559 & $<0.001$ & -0.329 & 0.038 \\
\hline \multicolumn{5}{|l|}{ HS evaluation } \\
\hline FLI & -0.762 & $<0.001$ & -0.633 & $<0.001$ \\
\hline
\end{tabular}

Correlations among variables were performed using Spearman's rho correlation coefficients. Circulating levels of Sirt4 were significantly associated with BMI, WC, metabolic profile, and the metabolic indices. After adjusting for BMI, the associations between circulating levels of Sirt 4 and the study variables were not significant, except for WC, HDL cholesterol, VAI, and FLI. A $p$ value in bold type denotes a significant difference $(p<0.05)$. Sirt4: sirtuin 4; BMI: body mass index; WC: waist circumference; SBP: systolic blood pressure; DBP: diastolic blood pressure; HDL: high-density lipoprotein; LDL: low-density lipoprotein; ALT: alanine aminotransferase; AST: aspartate aminotransferase; $\gamma$ GT: gamma glutamyl transferase; HoMA-IR: homeostatic model assessment-insulin resistance; VAI: visceral adiposity index; HS: hepatic steatosis; FLI: fatty liver index.

TABLE 6: Multinomial logistic regression of circulating levels of Sirt4 with HS grade.

\begin{tabular}{lccc}
\hline HS & OR & $p$ value & $95 \%$ IC \\
\hline Grade 1 = light & 9.47 & $\mathbf{0 . 0 0 2}$ & $7.02-12.77$ \\
Grade 2 = moderate & 3.22 & $\mathbf{0 . 0 0 5}$ & $2.39-4.35$ \\
Grade 3 = severe & 1.00 & $\mathbf{0 . 0 0 3}$ & $0.74-1.34$ \\
\hline
\end{tabular}

Multinomial logistic regression of circulating levels of Sirt4 with HS grade. The highest levels of Sirt4 were associated with the lowest odds of HS (grade 1). A $p$ value in bold type denotes a significant difference $(p<0.05)$. Sirt4: sirtuin 4; HS: hepatic steatosis; OR: odds ratio; IC: interval confidence.

obese individuals. In particular, the classification of patients according to the degree of adherence to the MD demonstrated that subjects with an average adherence presented a lower reduction in circulating levels of Sirt 4 when compared to subjects with a low adherence, independently of age and gender, with a PREDIMED score $\geq 6$ predicting the lowest decrease in circulating levels of Sirt4. Next, the study demonstrated that the highest consumption of food items characteristic of the
$\mathrm{MD}$, such as fruits, extra virgin olive oil, vegetables, and fish, was associated with a lowest decrease in circulating levels of Sirt4. The positive association between adherence to the MD and circulating levels of Sirt4 is consistent with the dietinduced modifications in Sirt1 [31] in the larger context of the diet-induced, epigenetic mechanisms [32]. This observation could be of potential interest considering the emerging role of Sirt4 as tumour suppressor recently reported in different human tumour cell lines [33] and the well-known antitumour properties of the $\operatorname{MD}[34,35]$.

The 7-day reports allowed us to obtain detailed information on the diet composition in subjects with low and average adherence to the MD. As expected, we found a highly significant correlation between PREDIMED score and MAI, an index developed to assess how close a diet is to the Healthy Reference National Mediterranean Diet [36]. When compared with average adherers, low adherers had a higher total energy intake, higher consumption of fat, mainly SFA and n-6 PUFA, and lower consumption of complex carbohydrate, protein, MUFA, n-3 PUFA, and fiber, and lower INQ for macronutrients. Consequently, low adherers had a low intake of all antioxidants considered in this study. Of 
TABLE 7: Bivariate proportional odds ratio model to assess the association between circulating levels of Sirt 4 and food items included in the PREDIMED questionnaire.

\begin{tabular}{|c|c|c|c|c|c|}
\hline Questions & OR & $p$ value & $95 \%$ IC & AIC & $R^{2}$ adj \\
\hline \multicolumn{6}{|c|}{ (1) Use of extra virgin olive oil as main culinary lipid } \\
\hline Yes & 2.60 & 0.004 & $1.93-3.51$ & 39.484 & 0.239 \\
\hline No & 1.04 & & $0.77-1.40$ & & \\
\hline \multicolumn{6}{|l|}{ (2) Extra virgin olive oil $>4$ tablespoons } \\
\hline Yes & 0.74 & 0.234 & $0.55-1.00$ & 62.069 & 0.254 \\
\hline No & 3.65 & & $2.70-4.92$ & & \\
\hline \multicolumn{6}{|l|}{ (3) Vegetables $\geq 2$ servings/day } \\
\hline Yes & 2.61 & 0.008 & $1.93-3.52$ & 53.157 & 0.175 \\
\hline No & 1.03 & & $0.77-1.44$ & & \\
\hline \multicolumn{6}{|l|}{ (4) Fruits $\geq 3$ servings/day } \\
\hline Yes & 3.44 & 0.002 & $2.55-4.63$ & 46.918 & 0.265 \\
\hline No & 0.78 & & $0.58-1.06$ & & \\
\hline \multicolumn{6}{|l|}{ (5) Red/processed meats $<1 /$ day } \\
\hline Yes & 1.33 & 0.246 & $0.98-1.79$ & 62.15 & 0.024 \\
\hline No & 2.04 & & $1.51-2.75$ & & \\
\hline \multicolumn{6}{|l|}{ (6) Butter, cream, margarine $<1 /$ day } \\
\hline Yes & 0.35 & 0.015 & $0.26-0.43$ & 52.652 & 0.175 \\
\hline No & 7.74 & & $5.74-10.44$ & & \\
\hline \multicolumn{6}{|l|}{ (7) Soda drinks $<1 /$ day } \\
\hline Yes & 1.14 & 0.593 & $0.85-1.54$ & 50.37 & 0.058 \\
\hline No & 2.36 & & $1.75-3.19$ & & \\
\hline \multicolumn{6}{|l|}{ (8) Wine glasses $\geq 7 /$ week } \\
\hline Yes & 2.41 & 0.018 & $1.78-3.25$ & 54.708 & 0.140 \\
\hline No & 1.12 & & $0.83-1.52$ & & \\
\hline \multicolumn{6}{|l|}{ (9) Legumes $\geq 3 /$ week } \\
\hline Yes & 1.65 & 0.067 & $1.23-2.23$ & 59.615 & 0.066 \\
\hline No & 1.63 & & $1.21-2.20$ & & \\
\hline \multicolumn{6}{|l|}{ (10) Fish/seafood $\geq 3 /$ week } \\
\hline Yes & 3.36 & 0.011 & $2.49-4.53$ & 50.762 & 0.200 \\
\hline No & 0.80 & & $0.59-1.08$ & & \\
\hline \multicolumn{6}{|c|}{ (11) Commercial sweets and confectionery $\leq 2 /$ week } \\
\hline Yes & 1.53 & 0.095 & $1.13-2.06$ & 52.011 & 0.057 \\
\hline No & 1.77 & & $1.31-2.39$ & & \\
\hline \multicolumn{6}{|l|}{ (12) Tree nuts $\geq 3 /$ week } \\
\hline Yes & 1.35 & 0.220 & $1.00-1.82$ & 55.183 & 0.028 \\
\hline No & 2.01 & & $1.49-2.71$ & & \\
\hline \multicolumn{6}{|l|}{ (13) Poultry more than red meats } \\
\hline Yes & 1.45 & 0.146 & $1.07-1.96$ & 61.239 & 0.039 \\
\hline No & 1.86 & & $1.38-2.52$ & & \\
\hline \multicolumn{6}{|l|}{ (14) Use of sofrito sauce $\geq 2 /$ week } \\
\hline Yes & 1.56 & 0.107 & $1.15-2.10$ & 60.355 & 0.051 \\
\hline No & 1.74 & & $1.29-2.34$ & & \\
\hline \multicolumn{6}{|l|}{ PREDIMED score } \\
\hline Low adherence MD (score $\leq 5)$ & 3.02 & 0.010 & $2.24-4.08$ & 20.409 & 0.710 \\
\hline Average adherence MD $(6 \leq$ score $\leq 9)$ & 8.97 & & $1.21-6.65$ & & \\
\hline
\end{tabular}

Circulating levels of Sirt4 were associated with the highest odds of consumption fruits, extra virgin olive oil, vegetables, fish, and wine and with highest score of adherence of MD. On the contrary, the highest levels of Sirt4 were significantly associated with the lowest odds of consumption of butter. A $p$ value in bold type denotes a significant difference $(p<0.05)$. PREDIMED: PREvención con DIeta MEDiterránea; OR: odds ratio; IC: interval confidence; AIC: Akaike information criterion. 
TABLE 8: Correlations of circulating levels of Sirt4 with PREDIMED score, total energy, and daily macronutrient/micronutrient intake.

\begin{tabular}{|c|c|c|c|c|}
\hline \multirow{3}{*}{ Parameters } & \multicolumn{4}{|c|}{$\begin{array}{l}\text { Circulating levels of Sirt4 (ng/mL) } \\
\qquad n=43\end{array}$} \\
\hline & \multicolumn{2}{|c|}{ Simple correlation } & \multicolumn{2}{|c|}{ Adjusted for total energy (kcal) } \\
\hline & $r$ & $p$ value & $r$ & $p$ value \\
\hline PREDIMED score & 0.820 & $<0.001$ & 0.356 & 0.021 \\
\hline Total energy (kcal) & -0.818 & $<0.001$ & - & - \\
\hline Protein (\% of total kcal) & 0.511 & $<0.001$ & 0.100 & 0.529 \\
\hline INQ protein & 0.742 & $<0.001$ & 0.453 & 0.003 \\
\hline Carbohydrate (\% of total kcal) & -0.070 & 0.656 & -0.033 & 0.838 \\
\hline INQ carbohydrate & 0.661 & $<0.001$ & 0.184 & 0.242 \\
\hline Complex (\% of total kcal) & 0.395 & 0.009 & 0.082 & 0.606 \\
\hline Simple (\% of total kcal) & -0.472 & 0.001 & -0.133 & 0.477 \\
\hline Fat (\% of total kcal) & -0.822 & $<0.001$ & -0.280 & 0.073 \\
\hline INQ Fat & 0.578 & $<0.001$ & 0.060 & 0.704 \\
\hline SFA (\% of total kcal) & -0.730 & $<0.001$ & -0.370 & 0.016 \\
\hline MUFA (\% of total kcal) & 0.739 & $<0.001$ & 0.138 & 0.384 \\
\hline PUFA (\% of total kcal) & -0.124 & 0.430 & 0.050 & 0.754 \\
\hline n-6 PUFA (g/day) & -0.546 & $<0.001$ & -0.107 & 0.502 \\
\hline n-3 PUFA (g/day) & 0.854 & $<0.001$ & 0.646 & $<0.001$ \\
\hline n-6/n-3 PUFAs ratio & -0.541 & $<0.001$ & 0.123 & 0.438 \\
\hline Cholesterol (mg/day) & -0.336 & 0.028 & -0.007 & 0.964 \\
\hline Fiber (g/day) & 0.305 & 0.047 & -0.026 & 0.872 \\
\hline Retinol Eq ( $\mu \mathrm{g} /$ day) & 0.337 & 0.027 & -0.029 & 0.854 \\
\hline Vitamin C (mg/day) & 0.825 & $<0.001$ & 0.476 & 0.001 \\
\hline Vitamin D ( $\mu \mathrm{g} /$ day $)$ & 0.407 & 0.007 & 0.083 & 0.601 \\
\hline Vitamin E (mg/day) & 0.834 & $<0.001$ & 0.329 & 0.034 \\
\hline Iron (mg/day) & 0.335 & 0.028 & 0.067 & 0.673 \\
\hline Selenium ( $\mu \mathrm{g} /$ day) & 0.421 & 0.005 & 0.205 & 0.192 \\
\hline Zinc (mg/day) & 0.505 & 0.001 & 0.020 & 0.900 \\
\hline MAI & 0.833 & $<0.001$ & 0.435 & 0.004 \\
\hline
\end{tabular}

Correlations among variables were performed Spearman's rho correlation coefficients. Circulating levels of Sirt4 were significantly associated with all the macronutrients/micronutrients evaluated in this study, except with carbohydrate and n-6 PUFA. After adjusting for total energy intake, the associations between circulating levels of Sirt4 and the study variables were lost, except for PREDIMED score, the consumption of fat, SFA and n-3 PUFA, and the intake of vitamins C and E. Sirt4: sirtuin 4; PREDIMED: PREvención con DIeta MEDiterránea; INQ: index of nutritional quality; SFA: saturated fatty acids; MUFA: monounsaturated fatty acids; PUFA: polyunsaturated fatty acids; MAI: Mediterranean adequacy index.

interest, after adjusting these results for total energy intake, circulating levels of Sirt4 remained significantly and positively associated only with the consumption of nutrients with the highest antioxidant properties, such as n-3 PUFA and vitamins $\mathrm{C}$ and $\mathrm{E}$, and negatively correlated with the consumption of SFA. The positive association of Sirt4 levels with dietary antioxidant intake and the negative association with VAI might be of particular interest considering the involvement of visceral adiposity in low-grade inflammation $[7,8]$ and the well-described effects of MD in inflammatory gene expression [37]. Using the multivariate analysis model, circulating levels of Sirt4 were well predicted by the PREDIMED score and the consumption n-3 PUFA.

As expected, obese subjects with a low adherence to MD presented worse anthropometric measurements and metabolic profile. In addition, as expected, low adherence to MD was associated to a higher HS grade when compared with subjects with an average adherence, independently of age and gender. Besides HS, this study reported a novel association between adherence to the MD and circulating levels of Sirt4 with VAI, a well validated, reliable, and widely used marker of ectopic visceral distribution and function [28]. In particular, VAI was higher in low adherers compared with average adherers and showed a negative correlation with circulating levels of Sirt4.

Taking together the results of this study: (i) lend support to the hypothesis that the increase in energy intake in obese individuals was associated with blunted circulating levels of Sirt4 as an adaptive mechanism to decrease fat oxidative capacity, but promoting lipid storage in ectopic tissues; (ii) suggest that the healthy combination of antioxidant vitamins and minerals characteristic of the MD could contribute to dampen this mechanism, probably reducing the oxidative damage and the ectopic fat storage. In line with this 
TABLE 9: Multiple regression analysis models (stepwise method) with the circulating levels of Sirt4 as dependent variable to estimate the predictive value of the following: (a) food items of PREDIMED questionnaire and PREDIMED score and (b) total energy intake, SFA, n-3 PUFA, vitamin C, and vitamin E.

\begin{tabular}{lcccc}
\hline Parameters & \multicolumn{5}{c}{ Multiple regression analysis } \\
\hline Model 1 & $R^{2}$ & $\beta$ & $t$ & $p$ value \\
PREDIMED score & 0.335 & 0.579 & 4.6 & $<\mathbf{0 . 0 0 1}$ \\
Fruits $\geq 3$ servings/day & 0.442 & 0.368 & 2.8 & $\mathbf{0 . 0 0 9}$ \\
Variable excluded: other & items of & PREDIMED score & \\
\hline Model 2 & $R^{2}$ & $\beta$ & $t$ & $p$ value \\
n-3 PUFA & 0.729 & 0.854 & 10.5 & $<\mathbf{0 . 0 0 1}$ \\
Vitamin E & 0.835 & 0.461 & 5.1 & $<\mathbf{0 . 0 0 1}$ \\
Vitamin C & 0.851 & 0.241 & 2.0 & $\mathbf{0 . 0 4 8}$ \\
Variable excluded: total energy and SFA & & \\
\hline
\end{tabular}

Among adherence to the MD and food items (model 1) and among dietary macronutrient and micronutrient intake (model 2), circulating levels of Sirt 4 were well predicted by PREDIMED score and $n-3$ PUFA. A $p$ value in bold type denotes a significant difference $(p<0.05)$. Sirt4: sirtuin 4 ; PREDIMED: PREvención con DIeta MEDiterránea; SFA: saturated fatty acids; PUFA: polyunsaturated fatty acids.

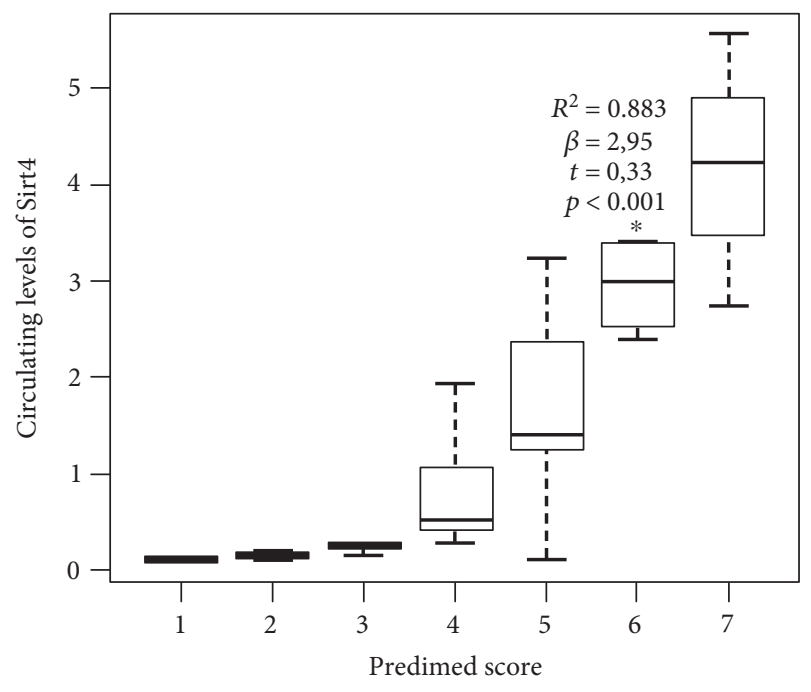

FIgURE 2: Box plot of value of the ordinal predictors between PREDIMED score and circulating levels of Sirt4. Based on the multiple linear regression analysis with ordinal predictors, the most significant $(\alpha=0.05)$ threshold value of the PREDIMED score predicting the lowest decrease in circulating levels of Sirt 4 was found at a score of $6\left({ }^{*} p<0.001\right)$. Sirt4: sirtuin 4; PREDIMED: PREvención con DIeta MEDiterránea.

hypothesis, circulating levels of Sirt 4 were well predicted by either the adherence to the MD and the intake of specific nutrients with antioxidant properties, independently of the energy intake; (iii) let us hypothesize that circulating levels of Sirt4, similarly to Sirt1, might undergo dietinduced epigenetic modifications, thus contributing to the antitumour properties of healthy diets, such as MD; and (iv) evidence that the lowest circulating levels of Sirt4 were associated not only to the highest grade of HS but also to the highest visceral fat dysfunction, independently of the severity of the obesity and physical activity.

Despite these very interesting results, the main limitation of this study is that the cross-sectional design does not allow to identify any causal association between the variables included. Second, we are aware that the sample size is small and the BMI range is wide and is not a measurement of body composition [38]. Nevertheless, we found that circulating levels of Sirt4 were negatively correlated to VAI, an index that incorporates also waist circumference, a far more accurate benchmark for visceral adiposity, also independently of BMI. Third, we do not provide experimental data on muscle biopsy sample between tissue expression and circulating levels of Sirt4; thus, our hypothesis of a compensative regulation of Sirt 4 by diet remains speculative. Furthermore, the suggested value of the PREDIMED score for the recognition of the lowest decrease in circulating levels of Sirt 4 should be viewed with caution until results will be extended to larger patient populations in order to perform an appropriate cross-validation. However, this study has adequate statistical power, the observed differences between low and average adherers were statistically significant, and correlations have been adjusted for BMI. In addition, in order to improve the power of the study, very stringent inclusion criteria have been applied, such as normal thyroid function or, according to our recent observations [39], normal somatotropic axis. Finally, the strength of this study is the usage of the 7-day food records. Although one week may not be representative of their usual dietary habits, this method of dietary assessment is considered as the "gold standard" in validation studies of different types of self-administered food frequency questionnaires, allowing a more accurate measurement of the real dietary and macronutrient intakes compared to those obtained by retrospective food frequency questionnaires [40, 41]. In addition, to reduce the effect of over- or under-reporting of individual foods, nutrient intake was adjusted for total energy intake.

Thus, although the results were partially limited due to the lack of specific markers of oxidative stress, the detailed information on the diet composition obtained by the 7-day food records was consistent with a more reduced intake of antioxidant nutrients in low adherers compared with average adherers.

4.1. Conclusions. A lower reduction in circulating levels of Sirt 4 was found in obese individuals with average adherence to the MD compared with low adherers, in association with lower ectopic fat storage and adipocyte dysfunction, independently of BMI. Circulating levels of Sirt4 varied in association not only with energy intake but also with antioxidant nutrient intake. The positive association between the adherence to the MD and circulating levels of Sirt4 suggests that the Sirt4 increase might represent further aspects of the antioxidant and antitumor advantage in obese individuals of the Mediterranean dietary pattern and support the role of qualified nutritionists as members of integrated multidisciplinary teams in the complex management of obesity. 


\section{Abbreviations}

$\begin{array}{ll}\text { ALT: } & \text { Alanine aminotransferase } \\ \text { AST: } & \text { Aspartate aminotransferase } \\ \text { BMI: } & \text { Body mass index } \\ \text { DBP: } & \text { Diastolic blood pressure } \\ \text { HDL: } & \text { High-density lipoprotein } \\ \text { HoMA-IR: } & \text { Homeostatic model assessment-insulin } \\ & \text { resistance } \\ \text { HS: } & \text { Hepatic steatosis } \\ \text { INQ: } & \text { Index of nutritional quality } \\ \text { LDL: } & \text { Low-density lipoprotein } \\ \text { MAI: } & \text { Mediterranean adequacy index } \\ \text { MD: } & \text { Mediterranean diet } \\ \text { MUFA: } & \text { Monounsaturated fatty acids } \\ \text { PREDIMED: } & \text { PREvención con DIeta MEDiterránea } \\ \text { PUFA: } & \text { Polyunsaturated fatty acids } \\ \text { RI: } & \text { Recommended intake } \\ \text { ROS: } & \text { Reactive oxygen species } \\ \text { SBP: } & \text { Systolic blood pressure } \\ \text { SFA: } & \text { Saturated fatty acids } \\ \text { Sirt4: } & \text { Sirtuin 4 } \\ \text { TG: } & \text { Triglycerides } \\ \text { VAI: } & \text { Visceral adiposity index } \\ \text { WC: } & \text { Waist circumference } \\ \gamma \text { GT: } & \text { Gamma glutamyl transferase. } \\ & \end{array}$

\section{Conflicts of Interest}

The authors declare that they have no conflict of interest.

\section{Authors' Contributions}

The authors' responsibilities were as follows: Luigi Barrea, Giovanni Tarantino, Carolina Di Somma, Andrea Falco, Giovanna Muscogiuri, and Silvia Savastano designed the research, conducted the research, wrote the paper, and had primary responsibility for the final content. Giovanna Muscogiuri and Paolo Emidio Macchia provided essential materials. Andrea Falco and Luigi Barrea analyzed the data and performed statistical analysis. All authors have read and approved the final manuscript. All authors contributed to and agreed on the final version of the manuscript.

\section{Acknowledgments}

This work has been partially supported by a grant from the Italian Ministry for University and Research (PRIN 2012 to Paolo Emidio Macchia). The authors would like to thank Antonio Improta, Dr. Giuseppe Annunziata, Dr. Lidia Albanese, and Dr. Vincenza Grazia Mele for data retrieval.

\section{References}

[1] M. A. Martínez-González, J. Salas-Salvadó, R. Estruch et al., "Benefits of the Mediterranean diet: insights from the PREDIMED study," Progress in Cardiovascular Diseases, vol. 58, no. 1, pp. 50-60, 2015.

[2] E. Scoditti, C. Capurso, A. Capurso, and M. Massaro, "Vascular effects of the Mediterranean diet-part II: role of omega-3 fatty acids and olive oil polyphenols," Vascular Pharmacology, vol. 63, no. 3, pp. 127-134, 2014.

[3] Z. Papackova and M. Cahova, "Fatty acid signaling: the new function of intracellular lipases," International Journal of Molecular Sciences, vol. 16, no. 2, pp. 3831-3855, 2015.

[4] M. T. Mitjavila, M. Fandos, J. Salas-Salvadó et al., “The Mediterranean diet improves the systemic lipid and DNA oxidative damage in metabolic syndrome individuals. A randomized, controlled, trial," Clinical Nutrition, vol. 32, no. 2, pp. 172-178, 2013.

[5] S. Marchi, C. Giorgi, J. M. Suski et al., "Mitochondria-ros crosstalk in the control of cell death and aging," Journal of Signal Transduction, vol. 2012, Article ID 329635, p. 17, 2012.

[6] M. S. Sozio, S. Liangpunsakul, and D. Crabb, "The role of lipid metabolism in the pathogenesis of alcoholic and nonalcoholic hepatic steatosis," Seminars in Liver Disease, vol. 30, no. 4, pp. 378-390, 2010.

[7] M. C. Amato, C. Giordano, M. Galia et al., "Visceral adiposity index: a reliable indicator of visceral fat function associated with cardiometabolic risk," Diabetes Care, vol. 33, no. 4, pp. 920-922, 2010.

[8] M. C. Amato, C. Giordano, M. Pitrone, and A. Galluzzo, "Cutoff points of the visceral adiposity index (VAI) identifying a visceral adipose dysfunction associated with cardiometabolic risk in a Caucasian Sicilian population," Lipids in Health and Disease, vol. 10, no. 1, p. 183, 2011.

[9] S. Petta, M. C. Amato, V. Di Marco et al., "Visceral adiposity index is associated with significant fibrosis in patients with non-alcoholic fatty liver disease," Alimentary Pharmacology \& Therapeutics, vol. 35, no. 2, pp. 238-247, 2012.

[10] T. T. Schug and X. Li, "Sirtuin 1 in lipid metabolism and obesity," Annals of Medicine, vol. 43, no. 3, pp. 198-211, 2011.

[11] S. Dato, D. Bellizzi, G. Rose, and G. Passarino, "The impact of nutrients on the aging rate: a complex interaction of demographic, environmental and genetic factors," Mechanisms of Ageing and Development, vol. 154, pp. 49-61, 2016.

[12] R. A. Mathias, T. M. Greco, A. Oberstein et al., "Sirtuin 4 is a lipoamidase regulating pyruvate dehydrogenase complex activity," Cell, vol. 159, no. 7, pp. 1615-1625, 2014.

[13] J. D. Covington and S. Bajpeyi, "The sirtuins: markers of metabolic health," Molecular Nutrition \& Food Research, vol. 60, no. 1, pp. 79-91, 2016.

[14] R. H. Houtkooper and J. Auwerx, "Exploring the therapeutic space around $\mathrm{NAD}^{+}$," The Journal of Cell Biology, vol. 199, no. 2, pp. 205-209, 2012.

[15] E. Pirinen, G. Lo Sasso, and J. Auwerx, "Mitochondrial sirtuins and metabolic homeostasis," Best Practice \& Research. Clinical Endocrinology \& Metabolism, vol. 26, no. 6, pp. 759-770, 2012.

[16] G. Tarantino, C. Finelli, F. Scopacasa et al., "Circulating levels of sirtuin 4 , a potential marker of oxidative metabolism, related to coronary artery disease in obese patients suffering from NAFLD, with normal or slightly increased liver enzymes," Oxidative Medicine and Cellular Longevity, vol. 2014, Article ID 920676, p. 10, 2014.

[17] G. Mancia, G. De Backer, A. Dominiczak et al., "Guidelines for the management of arterial hypertension: the task force for the management of arterial hypertension of the European Society of Hypertension (ESH) and of the European Society of Cardiology (ESC)," European Heart Journal, vol. 28, no. 12, pp. 1462-1536, 2007. 
[18] American Diabetes Association, "Standards of medical care in diabetes-2013," Diabetes Care, vol. 36, Supplement 1, pp. S11-S66, 2013.

[19] World Health Organ Tech Rep Ser, Obesity: Preventing and Managing the Global Epidemic. Report of a WHO Consultation No. 894, WHO, Geneva, Switzerland, 2000.

[20] Expert Panel on Detection, Evaluation, and Treatment of High Blood Cholesterol in Adults, "Executive summary of the third report of the National Cholesterol Education Program (NCEP) expert panel on detection, evaluation, and treatment of high blood cholesterol in adults (adult treatment panel III)," Jama, vol. 285, no. 19, pp. 2486-2497, 2001.

[21] M. A. Martínez-González, A. García-Arellano, E. Toledo et al., "A 14-item Mediterranean diet assessment tool and obesity indexes among high-risk subjects: the PREDIMED trial," PLoS One, vol. 7, no. 8, article e43134, 2012.

[22] L. Barrea, P. E. Macchia, G. Tarantino et al., "Nutrition: a key environmental dietary factor in clinical severity and cardiometabolic risk in psoriatic male patients evaluated by 7 -day food-frequency questionnaire," Journal of Translational Medicine, vol. 13, no. 1, p. 303, 2015.

[23] G. Turconi, M. Guarcello, F. G. Berzolari, A. Carolei, R. Bazzano, and C. Roggi, "An evaluation of a colour food photography atlas as a tool for quantifying food portion size in epidemiological dietary surveys," European Journal of Clinical Nutrition, vol. 59, no. 8, pp. 923-931, 2005.

[24] A. Alberti-Fidanza and F. Fidanza, "Mediterranean adequacy index of Italian diets," Public Health Nutrition, vol. 7, no. 07, pp. 937-941, 2004.

[25] A. Bach-Faig, E. M. Berry, D. Lairon et al., "Mediterranean diet pyramid today. Science and cultural updates," Public Health Nutrition, vol. 14, no. 12A, pp. 2274-2284, 2011.

[26] M. S. Won, S. Kim, and Y. J. Yang, "Comparison of health status and nutrient intake between depressed women and non-depressed women: based on the 2013 Korea National Health and Nutrition Examination Survey," Clinical Nutrition Research, vol. 5, no. 2, pp. 112-125, 2016.

[27] D. R. Matthews, J. P. Hosker, A. S. Rudenski, B. A. Naylor, D. F. Treacher, and R. C. Turner, "Homeostasis model assessment: insulin resistance and beta-cell function from fasting plasma glucose and insulin concentrations in man," Diabetologia, vol. 28, no. 7, pp. 412-419, 1985.

[28] M. C. Amato and C. Giordano, "Visceral adiposity index: an indicator of adipose tissue dysfunction," International Journal of Endocrinology, vol. 2014, Article ID 730827, p. 7, 2014.

[29] M. Webb, H. Yeshua, S. Zelber-Sagi et al., "Diagnostic value of a computerized hepatorenal index for sonographic quantification of liver steatosis," American Journal of Roentgenology., vol. 192, no. 4, pp. 909-914, 2009.

[30] E. Finell, U. Haverinen-Shaughnessy, A. Tolvanen et al., "The associations of indoor environment and psychosocial factors on subjective evaluation of indoor air quality among lower secondary school students - a multilevel analysis," Indoor air, vol. 27, no. 2, pp. 329-337, 2017.

[31] L. A. Wakeling, L. J. Ions, and D. Ford, "Could Sirt1-mediated epigenetic effects contribute to the longevity response to dietary restriction and be mimicked by other dietary interventions?" Age (Dordrecht, Netherlands), vol. 31, no. 4, pp. 327-341, 2009.

[32] S. J. van Dijk, P. L. Molloy, H. Varinli, J. L. Morrison, B. S. Muhlhausler, and Members of EpiSCOPE, "Epigenetics and human obesity," International Journal of Obesity, vol. 39, no. 1, pp. 85-97, 2015.

[33] S. M. Jeong, C. Xiao, L. W. Finley et al., "SIRT4 has tumor-suppressive activity and regulates the cellular metabolic response to DNA damage by inhibiting mitochondrial glutamine metabolism," Cancer Cell, vol. 23, no. 4, pp. 450-463, 2013.

[34] A. Giacosa, A. F. Adam-Blondon, S. Baer-Sinnott et al., "Alcohol and wine in relation to cancer and other diseases," European Journal of Cancer Prevention, vol. 21, no. 1, pp. 103-108, 2012.

[35] N. Di Daniele, A. Noce, M. F. Vidiri et al., "Impact of Mediterranean diet on metabolic syndrome, cancer and longevity," Oncotarget, vol. 8, no. 5, pp. 8947-8979, 2017.

[36] I. Castro-Quezada, B. Román-Viñas, and L. Serra-Majem, "The Mediterranean diet and nutritional adequacy: a review," Nutrients, vol. 6, no. 1, pp. 231-248, 2014.

[37] V. Konstantinidou, M. I. Covas, R. Sola, and M. Fitó, "Up-to date knowledge on the in vivo transcriptomic effect of the Mediterranean diet in humans," Molecular Nutrition \& Food Research, vol. 57, no. 5, pp. 772-783, 2013.

[38] J. C. Wells and M. S. Fewtrell, "Measuring body composition," Archives of Disease in Childhood, vol. 91, no. 7, pp. 612-617, 2006.

[39] S. Savastano, C. Di Somma, A. Colao et al., "Preliminary data on the relationship between circulating levels of Sirtuin 4 , anthropometric and metabolic parameters in obese subjects according to growth hormone/insulin-like growth factor-1 status," Growth Hormone \& IGF Research, vol. 25, no. 1, pp. 28-33, 2015.

[40] F. E. Thompson, Subar AF Chapter 1-Dietary Assessment Methodology. Nutrition in the Prevention and Treatment of Disease, Academic Press, Burlington, 2008.

[41] S. Høidrup, A. H. Andreasen, M. Osler et al., "Assessment of habitual energy and macronutrient intake in adults: comparison of a seven day food record with a dietary history interview," European Journal of Clinical Nutrition, vol. 56, no. 2, pp. 105-113, 2002. 


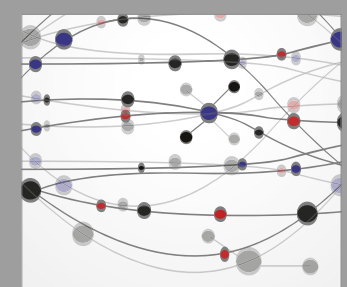

The Scientific World Journal
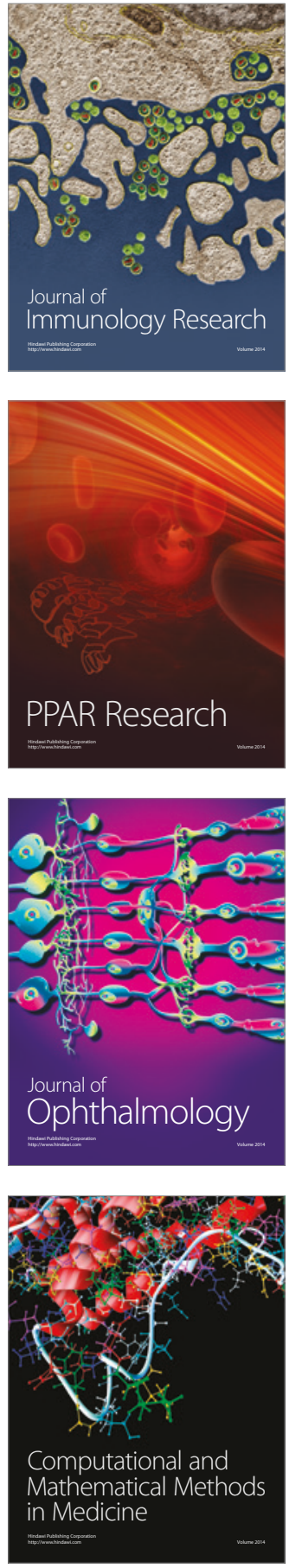

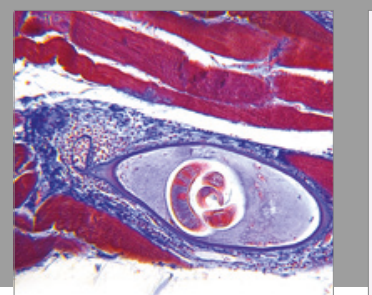

Gastroenterology Research and Practice
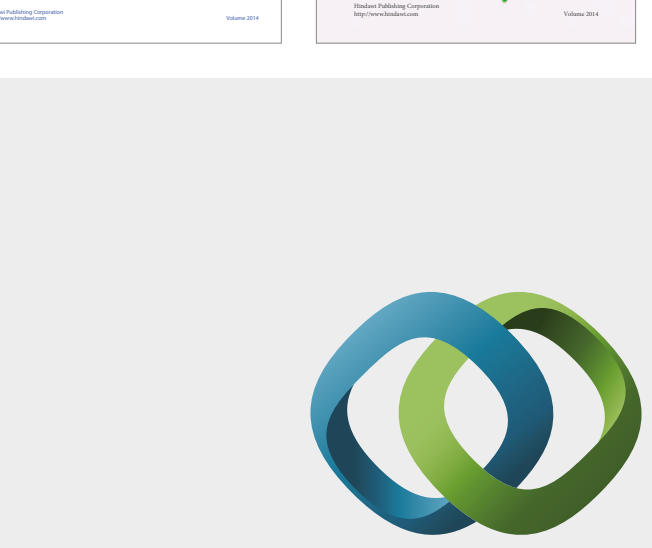

\section{Hindawi}

Submit your manuscripts at

https://www.hindawi.com
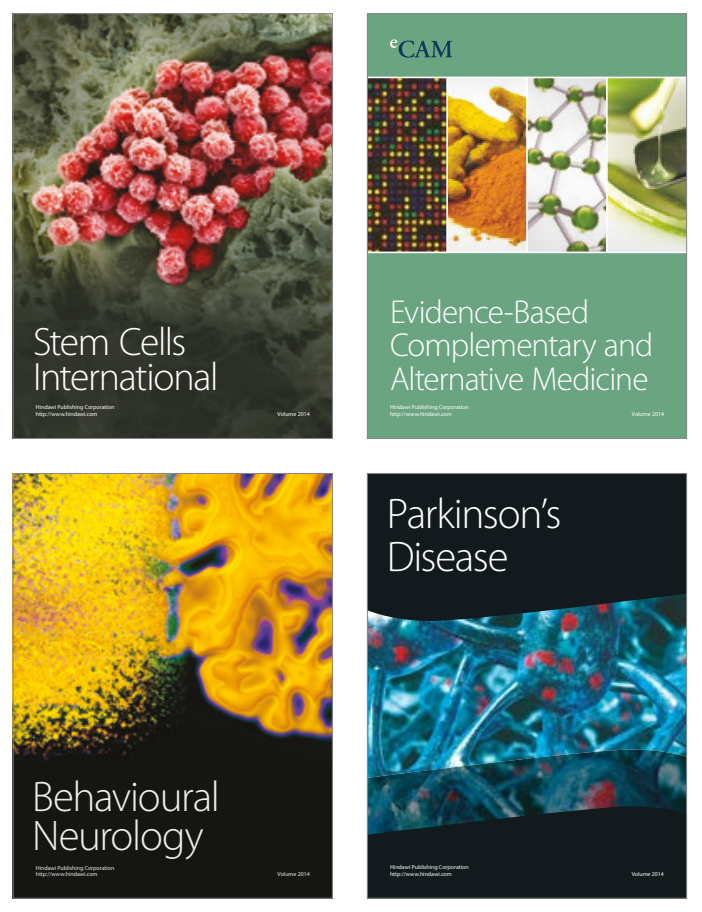
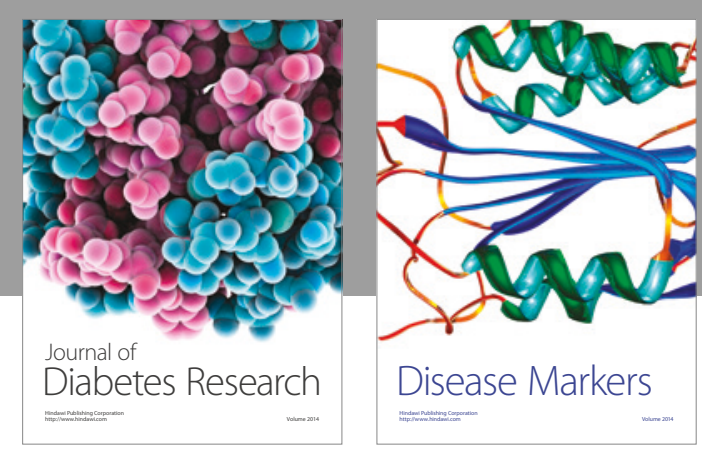

Disease Markers
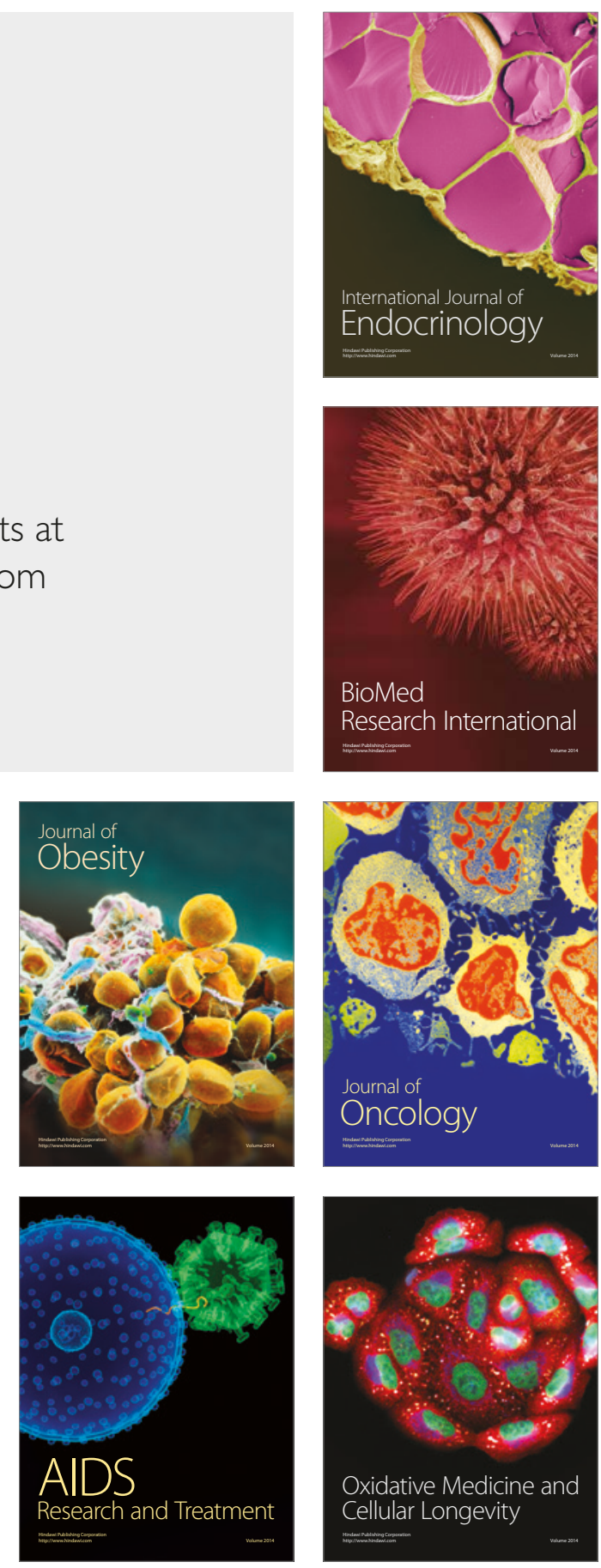